\title{
Pallidal neurostimulation versus botulinum toxin injections in the treatment of cervical dystonia: protocol of a randomized, sham- controlled trial (StimTox-CD)
}

Thorsten M. Odorfer ${ }^{1 *}$ (D) Uwe Malzahn², Cordula Matthies ${ }^{3}$, Peter U. Heuschmann²,4 and Jens Volkmann ${ }^{5}$

\begin{abstract}
Background: Selective peripheral denervation via botulinum neurotoxin injections into dystonic muscles is the first-line treatment for cervical dystonia. Pallidal neurostimulation is a potent alternative, but currently restricted to patients failing on neurotoxin therapy. As botulinum neurotoxin is partially effective but often unsatisfactory in a relevant proportion of patients, earlier neurostimulation might be advantageous in providing stable symptom control and preventing disability. This trial intends to demonstrate, that pallidal neurostimulation is superior to neurotoxin injections in best clinical practice for controlling the symptoms of cervical dystonia and that it is safe in patients with a partial therapy response to peripheral denervation. We hypothesize a better outcome in everyday functioning and health-related quality of life of neurostimulated patients.
\end{abstract}

Methods: We aim to recruit 66 cervical dystonia patients into a double-blind comparison of pallidal neurostimulation versus botulinum neurotoxin type A. Eligible patients need $\geq 25 \%$ motor symptom reduction 4 weeks after a neurotoxin test injection, but are willing to undergo DBS surgery due to unsatisfactory symptom control. All participants will be implanted with a DBS system, and randomized into 2 groups: First group will receive effective neurostimulation and saline injections into dystonic muscles. Second group is treated with regular neurotoxin injections and undergoes a sham-stimulation. Primary outcome is the change in TWSTRS total score between baseline and 6 months of therapy. Secondary outcome parameters are corresponding changes in TWSTRS motor score, Tsui score, CDQ-24 and SF-36. Safety will be assessed by frequency and severity of reported adverse events. Statistical analysis includes intention-to-treat and per protocol populations, analysis based on imputation of missing values and analysis adjusting for differences in baseline TWSTRS. After 6 months of blinded treatment all patients will receive open-label neurostimulation and neurotoxin treatment as needed, and are followed up 48 weeks after randomization.

Perspective: We will assess if pallidal neurostimulation is a safe and effective alternative to selective peripheral denervation by botulinum toxin injections in cervical dystonia, which may be offered earlier in the course of disease based on patient preference. A positive study outcome would influence future treatment guidelines of cervical dystonia.

Trial registration: EudraCT registration number: 2016-001378-13

Keywords: Cervical dystonia (CD), Pallidal neurostimulation, Deep brain stimulation (DBS), Botulinum neurotoxin type a (BoNT a), Motor symptom control, TWSTRS (Toronto Western spasmodic torticollis rating scale), dystonia-associated quality of life, Safety

\footnotetext{
* Correspondence: odorfer_T@ukw.de

${ }^{1}$ Department of Neurology, University Hospital Würzburg, Josef-Schneider-Str.

11, 97080 Würzburg, Germany

Full list of author information is available at the end of the article
}

(c) The Author(s). 2019 Open Access This article is distributed under the terms of the Creative Commons Attribution 4.0 International License (http://creativecommons.org/licenses/by/4.0/), which permits unrestricted use, distribution, and reproduction in any medium, provided you give appropriate credit to the original author(s) and the source, provide a link to the Creative Commons license, and indicate if changes were made. The Creative Commons Public Domain Dedication waiver (http://creativecommons.org/publicdomain/zero/1.0/) applies to the data made available in this article, unless otherwise stated. 


\section{Background}

Cervical dystonia (CD) is a central nervous system disease associated with involuntary muscle contractions, leading to repetitive or constant neck movements and partly bizarre head postures [1]. CD is estimated to be the most frequent of focal dystonias [9]. Activities of daily living, social participation and quality of life can be markedly impaired in $\mathrm{CD}$ due to pain, reduced active head and neck motion ranges as well as stigmatization (Camfield, Ben-Shlomo, Warner, \& Epidemiological Study of Dystonia in Europe Collaborative, $[4,15])$.

Chemical denervation via injection of botulinum neurotoxin (BoNT) into dystonic neck muscles is applied as first line treatment of $\mathrm{CD}$; its efficacy and safety has been demonstrated in several randomized controlled trials $[5,18]$. BoNT injections are shown to reduce mean symptom severity substantially defined as reduction between 9.7 to 11 points on Toronto Western Torticollis Rating Scale (TWSTRS) [2, 6]. In addition, its application is superior to pharmacological treatment with anticholinergic drugs [3]. As the neurotoxin effect is only temporary, repeated injections are necessary within a period of 8 to 16 weeks. Furthermore, the toxin effect builds up within a period of 1-4 weeks after injection; therefore, $\mathrm{CD}$ symptom severity tends to fluctuate during BoNT therapy. Beside these pharmacological response fluctuations, patients may experience a variable benefit from repeated injections, because the treatment is skill-dependent and limited by the reproducibility of applying equivalent toxin dosages into partly deep lying neck muscles. Beyond the principle efficacy established in pivotal trials, few studies have addressed the long-term efficacy and stability of BoNT treatment responses in daily practice. In an observational study Misra et al. reported that more than $60 \%$ of $C D$ patients are treated satisfactorily with BoNT; however, considering the criterion of "duration of effect $\geq 12$ weeks" reduced this proportion up to $29 \%$ [14]. A further limitation of BoNT therapy arises when complex dystonic patterns including tremor or jerky movements appear; in these cases, many muscles are involved in an intricate interplay, which apparently impedes a targetoriented injection. Additionally, dose-dependent adverse events (AE) may limit the treatment, such as neck weakness, dysphagia, dry mouth or dysphonia [7]. During chronic treatment, secondary therapy failure due to BoNT neutralizing antibodies develops in up to $5 \%$ of patients [10]. Despite these limitations and the discomfort associated with repeated BoNT injections, many patients adhere to this therapy, because they are not aware of potential alternatives.

Deep brain stimulation (DBS) is a surgical therapy for movement disorders, which retunes abnormal neuronal activity within motor circuits by continuous electrical stimulation of deep brain nuclei. DBS requires the surgical implantation of stimulating electrodes connected to a pacemaker device. There is class I evidence for the safety and efficacy of DBS applied to several brain targets (subthalamic nucleus, internal globus pallidus (GPi) and thalamus) for patients with Parkinson's disease (PD) [8], tremor disorders [17] and dystonia [12]. Treatment effects of DBS are strong and can lead to almost complete symptom suppression even in severely disabled patients. Nevertheless, this therapy has been restricted to advanced disease stages after failure of pharmacological treatment alternatives out of principle safety considerations. DBS surgery carries a risk of permanent morbidity and mortality resulting from intracranial haemorrhage or infections, while the treated conditions are disabling but not life limiting by themselves. Otherwise, DBS may come too late in advanced disease stages for restoring function and societal participation in chronically disabled patients despite being effective on a symptom level. Improved surgical techniques and perioperative management have now reduced the chronic morbidity and mortality risk of DBS below $0.5 \%$ [11], which has allowed considering earlier DBS with the goal to maintain function and prevent psychosocial decline in PD. For example, the EARLYSTIM trial [16], a controlled randomized comparison of subthalamic DBS with best medical management of PD with early motor fluctuations has established superiority of DBS for improving off-period symptoms, response fluctuations and quality of life. Interestingly, there was no difference in the total number of $\mathrm{AE}$ and serious $\mathrm{AE}$ between both treatment groups over the 2 year study period, suggesting that the risks associated with medical treatment or inappropriate symptom control of PD have been largely underestimated in the past and may be mitigated by DBS.

GPi-DBS is an established first line therapy for patients with generalized dystonia, for whom no pharmacological treatment alternatives exist [12, 19]. Previous trials have also established efficacy and safety in patients with $C D$ refractory to neurotoxin therapy. In a severely affected study population, a mean reduction of 5.2 points $(26 \%)$ on TWSTRS motor and 18.3 points (40\%) on TWSTRS total score was achieved by GPi-DBS [19]. Additionally, also pain, activities of daily living and quality of life are shown to improve during DBS treatment (Kupsch et al., 2006; $[19,20])$.

Based on this evidence, we believe, that the acceptable safety profile and the therapeutic potency of DBS justify an earlier application in $\mathrm{CD}$ patients still responding to peripheral selective denervation, but suffering from incomplete symptom control or response fluctuations. In this trial we aim to prove that GPi-DBS leads to a superior symptom control in comparison to best clinical use of BoNT in the group of CD patients with a partial response to BoNT therapy. Consequently, we also hypothesize that dystonia-associated quality of life improves more with DBS treatment. Additionally, safety of DBS will be explored in this patient group. 


\section{Methods/design}

\section{Study protocol}

Figure 1 shows the study schedule of StimTox-CD. During "screening visit" (SCR) a test BoNT injection is applied to prove partial therapy response of neurotoxin treatment. $4( \pm 1)$ weeks later in "evaluation visit" (EVA) treatment effect is quantified by clinical assessment comprising inter alia TWSTRS motor score. Finally, a minimal motor-symptom reduction of $25 \%$ is required to fulfil all inclusion criteria. Table 1 shows a comprehensive list of the criteria applied to choose eligible patients for the study.

All participants undergo stereotactic implantation of deep brain electrodes in GPi under general anaesthesia. Comparable to previous dystonia DBS studies, the applied neurostimulation system is ACTIVA PC (MEDTRONIC, Minneapolis, USA), including matching electrodes 3387 and 3389 [13, 19]. DBS procedure depends on local standards in each study centre. There are no detailed specifications on perioperative management and stereotactic procedure. Only a preoperative MRI-scan (including T1-, T2, gadolinium-enhanced and inversion recovery sequences) in general anaesthesia and a postoperative image control of electrode positioning are mandatory.

$6( \pm 1)$ weeks after DBS implantation patients are divided in two study arms during "randomization visit" (RAN). Table 2 is showing the full data set, which is collected at RAN as baseline for the upcoming blinded study phase. Neurostimulation will be now activated for the first time and acute stimulation effects and adverse effects of all electrodes are tested in a structured fashion ("monopolar review"). In DBS group a local DBS expert implements an effective stimulation setting concerning stimulation amplitude and choice of electrodes after "best clinical practice" and a predetermined algorithm. Frequency must be adjusted to $130 \mathrm{~Hz}$, pulse duration to $90 \mu \mathrm{s}$. In BoNT group stimulation amplitude is set to $0 \mathrm{~V}$. This group is injected with BoNT by an experienced physician. The decision on preferred drugs (Onabotulinumtoxin A: BOTOX, Abobotulinumtoxin A: DYSPORT or Incobotulinumtoxin A: XEO$\mathrm{MIN}$ ), applied dosage and injection technique (number of injections, injection site or guiding-techniques) is up to the discretion of the treating physician and supposed to correspond to best clinical practice. During the same visit, patients in DBS group get also injected, but only with saline. Neither patient nor treating physician is informed about the allocation to the study group, in the sense of a double-blind trial design. Obviously DBS setting is not appropriate to be implemented in a blind manner, what causes the need of two separate teams of physicians at each study site; one blinded (or BoNT) and one unblinded (or DBS) team.

$4( \pm 1)$ weeks after randomization first follow-up visit (FU1) is scheduled. Clinical outcome parameters are evaluated by blinded physicians. The same procedure is repeated during FU2 and FU3 $(12( \pm 1)$ and $16( \pm 1)$ weeks after RAN), with a second blindly applied BoNT or placebo injection. In FU2 also adjustments of DBS

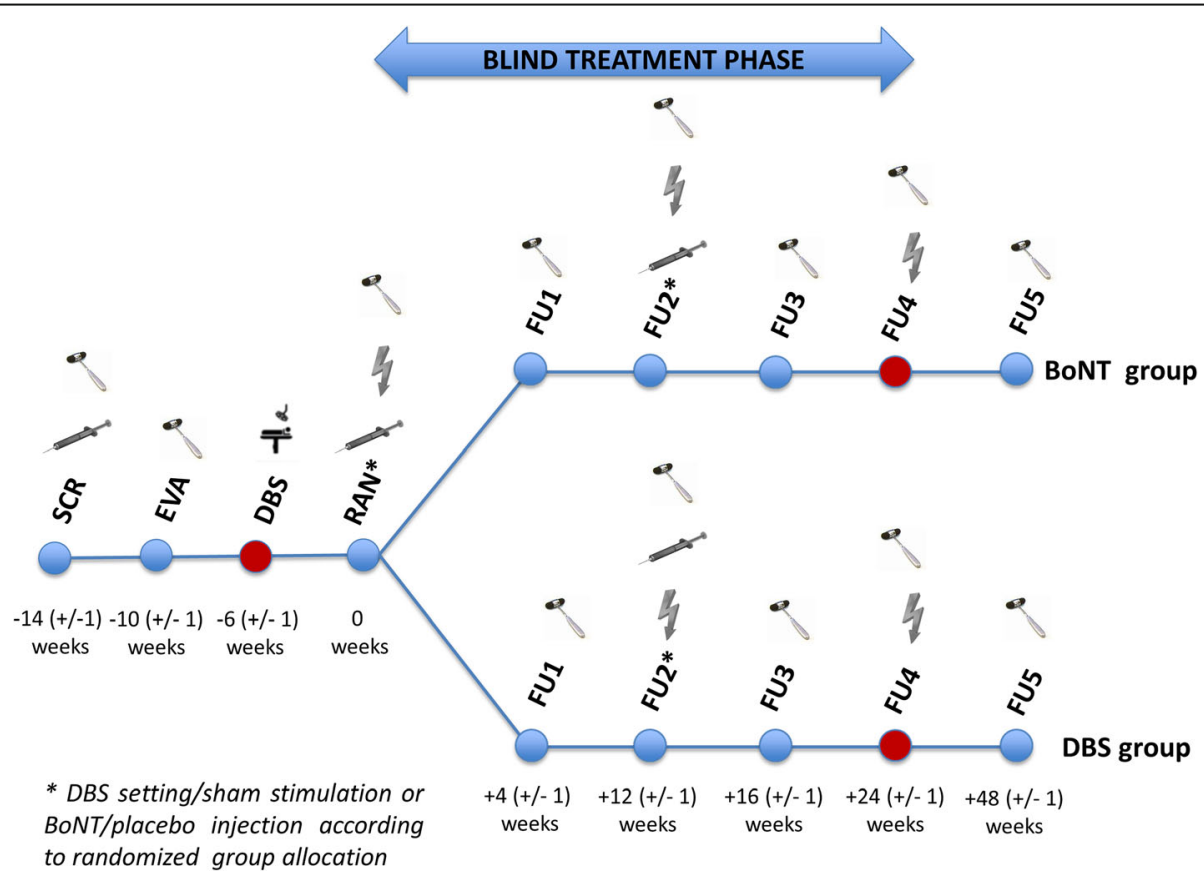

Fig. 1 Visit schedule of StimTox-CD: SCR = screening visit, EVA = evaluation visit, DBS = DBS surgery, RAN = randomization visit, FU = follow-up visit, icon "syringe" $\hat{=}$ BoNT or placebo injection, icon "reflex hammer" $\hat{=}$ clinical assessment, icon "operating room" $\hat{=}$ DBS surgery, icon "lightning" $\hat{=}$ DBS setting/sham stimulation 
Table 1 Eligibility criteria

\begin{tabular}{ll}
\hline Inclusion criteria & Exclusion criteria \\
\hline - Suffering from isolated, idiopathic or hereditary cervical dystonia for more & - Mild dystonic symptoms (TWSTRS total score $<10$ points and \\
than 2 years & TWSTRS motor score $<5$ points) \\
- Moderate to severe symptom severity of dystonia (TWSTRS total score & - Suffering from severe depression with ongoing suicidality \\
$\geq 20$ points and TWSTRS motor score $\geq 15$ points) & (BDI $>25$ points) \\
- Age between 18 and 75 years & - Mild cognitive impairment (MCI) and dementia (MDRS $\leq 125$ points) \\
- Adequate therapy response of BoNT A treatment during test injection & - Ongoing acute psychosis \\
procedure $(\geq 25 \%$ reduction of points in TWSTRS motor score 4 weeks & - Drug or alcohol abuse \\
after baseline) & - Pregnancy or lactation, women of childbearing age without secure \\
- Previous BoNT injection $\geq 12$ weeks before screening visit & contraception \\
- Written informed consent to study participation, including patient's & - Illiteracy \\
agreement to undergo DBS procedure & - Surgical contraindication to DBS \\
& - Concurrent participation in other clinical trials \\
& - Any medical or psychological condition associated with the risk of \\
& insufficient compliance and/or early termination of the study \\
\hline
\end{tabular}

settings are allowed to improve neurostimulation effect in DBS group. The blinded study phase ends in FU4, 24 $( \pm 1)$ weeks after randomization. Neurostimulation is now activated also in BoNT group and the open trial phase starts. Up from FU4, patients are treated commonly without restrictions concerning DBS programming or BoNT injection, treatment must basically conform again to best clinical practice. During FU5, after another interval of $24( \pm 1)$ weeks, study ends and a last detailed clinical assessment is intended.

\section{Outcome measures}

The primary outcome parameter is the difference in TWSTRS total score 6 month after implantation. We hypothesize that under optimized treatment in both groups there should be no discrepancy in symptom severity at FU3 (4 weeks after injection of BoNT at the time of medication's peak effect).

We defined changes in TWSTRS motor score, TSUI score, CDQ-24 and SF-36 to serve as secondary outcome parameters. Additionally, we measure frequency and severity of spontaneously reported AE in both treatment arms.

Additionally, we will describe the difference in TWSTRS total score between patients of DBS group at FU4 and BoNT group at FU5. At both time points, patients are treated with effective neurostimulation for 6 months, respectively. We suppose the same therapy response of effective DBS in both arms, regardless if the stimulation is applied in a blinded or unblinded fashion.

As other hypothesis we will investigate if the maximum treatment effect of DBS is achieved 6 month after activation. Therefore, we will quantify variations in TSWTRS score in the DBS group 6 and 12 months after randomization.

Motor symptom severity is basically evaluated by using standardized and well-established scales like TWSTRS motor and Tsui score. Tsui includes also a tremor item, which is missing in TWSTRS. Dystonic head tremor is also assessed by the Bain Tremor Rating Scale. Additionally, the severity of dystonia is ranked by patients and physicians using a numeric rating scale (1-10) as element of the Global Clinical Impression (GCI) scale.

Questionnaires referring to pain and disability are also part of TWSTRS total score. Another dystonia-specific tool applied to assess quality of life of patients suffering from cervical dystonia is the Craniocervical Dystonia Questionnaire (CDQ-24). Two further standardized forms supposed to measure general health-related quality of life were chosen to be completed by study participants: SF-36 (Short Form 36) and EQ-5D (Euroquol). To exclude relevant cognitive deficits Mattis Dementia Rating Scale (MDRS) will be applied. Depressive symptoms are assessed by Beck Depression Inventory (BDI). For other psychiatric comorbidities we screen by using the Brief Psychiatric Rating Scale (BPRS).

All scales and questionnaires are used in their German version.

\section{Randomization}

Patients are allocated in a 1:1 ratio into the two study arms by stratified block randomization. Randomization procedure is operated centrally via GCP-compliant EDC system. The categorized baseline TWSTRS total score serves as single stratification factor to aim approximately equal distribution of symptom severity in both groups.

\section{Statistics}

Sample size calculation is based on primary outcome parameter TWSTRS total score change from baseline to FU4. The primary statistical analysis consists in a mean value comparison of the primary outcome between both treatment groups by the independent two-sample T-Test. For sample size calculation we refer to data from previously published studies. A GPi-DBS trial showed a mean therapy effect in TWSTRS total score in patients undergoing neurostimulation of minus 23.1 ( \pm 15.2 SD) points [19]. A mean maximum effect of neurotoxin treatment is described with minus $11.0( \pm 11.7 \mathrm{SD})$ points on TWSTRS total score at an average of 4 weeks after injection [6]. The resulting standardized mean 
Table 2 Synopsis of interventions and data sets (displayed for single SimTox-CD study visits)

\begin{tabular}{|c|c|c|c|c|c|c|c|c|c|}
\hline & $\begin{array}{l}\text { Screening } \\
\text { visit/SCR }\end{array}$ & $\begin{array}{l}\text { Evaluation } \\
\text { visit/EVA }\end{array}$ & $\begin{array}{l}\text { DBS } \\
\text { surgery/DBS }\end{array}$ & $\begin{array}{l}\text { Randomization } \\
\text { visit/RAN }\end{array}$ & $\begin{array}{l}\text { Follow-up } \\
\text { visit 1/FU1 }\end{array}$ & $\begin{array}{l}\text { Follow-up } \\
\text { visit 2/FU2 }\end{array}$ & $\begin{array}{l}\text { Follow-up } \\
\text { visit 3/FU3 }\end{array}$ & $\begin{array}{l}\text { Follow-up } \\
\text { visit 4/FU4 }\end{array}$ & $\begin{array}{l}\text { Follow-up } \\
\text { visit 5/FU5 }\end{array}$ \\
\hline timeline (weeks) & $\begin{array}{l}-14 \\
(+/-1)\end{array}$ & $\begin{array}{l}-10 \\
(+/-1)\end{array}$ & $\begin{array}{l}-6 \\
(+/-1)\end{array}$ & 0 & $\begin{array}{l}+4 \\
(+/-1)\end{array}$ & $\begin{array}{l}+12 \\
(+/-1)\end{array}$ & $\begin{array}{l}+16 \\
(+/-1)\end{array}$ & $\begin{array}{l}+24 \\
(+/-1)\end{array}$ & $\begin{array}{l}+48 \\
(+/-1)\end{array}$ \\
\hline \multicolumn{10}{|l|}{ Interventions } \\
\hline BoNT test injection & $x$ & & & & & & & & \\
\hline DBS surgery & & & $x$ & & & & & & \\
\hline $\begin{array}{l}\text { Testing of stimulation (side) } \\
\text { effects }\end{array}$ & & & & $x$ & & & & & \\
\hline DBS setting/sham stimulation ${ }^{a}$ & & & & $x$ & & $x$ & & & \\
\hline Blind BoNT/placebo injection ${ }^{a}$ & & & & $x$ & & $x$ & & & \\
\hline Unblind neurostimulation & & & & & & & & $x$ & $x$ \\
\hline Randomization & & & & $x$ & & & & & \\
\hline \multicolumn{10}{|l|}{ Data set } \\
\hline Informed consent & $x$ & & & & & & & & \\
\hline Inclusion/exclusion criteria & $x$ & $x$ & & & & & & & \\
\hline Medical history & $x$ & & & & & & & & \\
\hline Concurrent medication & $x$ & & & & $x$ & $x$ & $x$ & $x$ & $x$ \\
\hline TWSTRS & $x$ & $x$ & & $x$ & $x$ & $x$ & $x$ & $x$ & $x$ \\
\hline Tsui Rating Scale & $x$ & $x$ & & $x$ & $x$ & $x$ & $x$ & $x$ & $x$ \\
\hline Bain Tremor Rating & $x$ & $x$ & & $x$ & $x$ & $x$ & $x$ & $x$ & $x$ \\
\hline $\mathrm{GCl}$ & $x$ & $x$ & & $x$ & $x$ & $x$ & $x$ & $x$ & $x$ \\
\hline CDQ-24 & $x$ & & & $x$ & & & & $x$ & $x$ \\
\hline SF-36 & $x$ & & & $x$ & & & & $x$ & $x$ \\
\hline EQ-5D & $x$ & & & $x$ & & & & $x$ & $x$ \\
\hline MDRS & $x$ & & & $x$ & & & & $x$ & $(X)$ \\
\hline BDI & $x$ & & & $x$ & & & & $x$ & $(X)$ \\
\hline BPRS & $x$ & & & $x$ & & & & $x$ & $(X)$ \\
\hline Video recording & $(X)$ & $(X)$ & & $x$ & $(X)$ & $(X)$ & $(X)$ & $x$ & $x$ \\
\hline $\begin{array}{l}\text { Questionnaire on } \\
\text { DBS procedure }\end{array}$ & & & $x$ & & & & & & \\
\hline $\begin{array}{l}\text { Postoperative control of } \\
\text { electrode position }\end{array}$ & & & & $x$ & & & & & \\
\hline$A E$ & & & & & $x$ & $x$ & $x$ & $x$ & $x$ \\
\hline DBS settings & & & & $x$ & $x$ & $x$ & $x$ & $x$ & $x$ \\
\hline
\end{tabular}

according to randomized group allocation

Abbreviations (in alphabetical order): $A E$ adverse events, BoNT Botulinum neurotoxin, BDI Beck Depression Inventory, BPRS Brief Psychiatric Rating Scale, CDQ-24 Craniocervical Dystonia Questionnaire, DBS deep brain stimulation, EQ-5D Euroquol (life quality questionnaire), GCI Global Clinical Impression Scale, MDRS Mattis Dementia Rating Scale, TWSTRS Toronto Western Spasmodic Torticollis Rating Scale, SF-36 Short Form 36 (life quality questionnaire)

difference of TWSTRS scores between both groups is 0.90. Using these assumptions a sample size of $2 \times 28=56$ patients ensures a power of $90 \%$ to detect this effect size as significant (at significance level 0.05) deviation from the null hypothesis (no difference between mean TWSTRS change scores in DBS and BoNT group). Taking into account a drop-out rate of $15 \%$ (conservative estimation) $2 \times 33=66$ must be randomized. Furthermore we assume that $20 \%$ of patients will refuse informed consent to undergo DBS after screening and another 20\% will fail inclusion criteria of sufficient botulinum treatment response after test injection. Consequently, a total number of $2 \times 52=$ 104 patients will have to be screened to reach the calculated sample size.

Group comparisons for target variables with sample distributions compatible with the assumption of normality will be conducted by using the two-sided independent sample Student's T-Test or the paired sample T-Test for within group comparisons (changes between visits). For variables with sample distributions not compatible with the 
assumption of normality non-parametric tests like Mann-Whitney-Test, Wilcoxon Signed Rank Test or Friedmann Test will be applied. Effects will be considered significant if $p<0.05$.

The primary statistical analysis of the primary outcome parameter as well as the secondary outcome parameter change in TWSTRS motor score, TSUI score and SF-36 score will be based on the intention-to-treat (ITT) population. To proof robustness of results two further analyses of sensitivity will be performed: the first one refers to all randomized patients with imputation of missing values after 6 months by appropriate methods of missing-data imputation depending on which missing-data mechanism could be reasonable. The second method includes only patients of per protocol (PP) population.

To address the question how much TWSTRS total score values differ between DBS and BoNT group on average, when patients would be start at baseline with the same score values, we will use ANCOVA (target variable: TWSTRS total change score from baseline to 6 months after operation, main factor: treatment method, covariate: baseline TWSTRS). Thereby we will adjust to imbalances in the TWSTRS distributions at baseline and the variance within the groups should be reduced.

Also for secondary outcome parameters an ANCOVA will be applied using treatment group as main factor, adjusted for each baseline variable as covariate. Furthermore longitudinal data of scores will be analysed by using GEE (generalized estimating equation) and linear models ("mixed models"). This will be helpful to describe interindividual variability of therapy response.

Safety analysis contains a structured list of AE of both study groups. Possibility of relation to study's interventions will be judged by physicians of the unblinded team. Incidence of AE will be assessed by using Fisher's Exact Test (analysis by patients: comparisons of proportions of patients with at least one corresponding event) and negative binomial regression (analysis by events: comparisons of counts of occurring events).

\section{Perspective}

StimTox-CD is the first multicentre, randomized, double-blind and sham-controlled trial of DBS vs. best conservative therapy, specifically selective peripheral denervation with BoNT in CD patients, which have a partial treatment effect of ongoing neurotoxin injections. The study hypothesizes that pallidal neurostimulation is more effective in controlling CD symptoms than BoNT treatment and that is safe in this indication, too.

\section{Visit schedule}

To provide a fair comparison between the two treatments with time varying efficacy, we took special care in scheduling the study visits. Baseline visit takes place 6 weeks after operation. This interval was chosen to reduce the impact of the surgical stun effect of electrode implantation. Additionally, motor symptom control efficacy is evaluated in FU1 and FU3 (each 4 weeks after BoNT injection), when the neurotoxin treatment effect is supposed to be maximal [6].

\section{Quality management}

In order to maintain comparable and highest treatment standards at all study sites, we only recruited DBS centres operating more than 25 patients within a year. All 11 participating sites are university hospitals in Germany with a longstanding experience in DBS and a BoNT outpatient clinic run by an expert physician (board certified neurologist and special experience in neurotoxin treatment).

Furthermore, we provide a controlled rating training for TWSTRS score to increase inter-rater-reliability. To reduce possible bias trough changing or different examiners, a standardized video recording is scheduled at RAN, FU4 and FU5 to enable a central evaluation of symptom severity.

\section{Blinding}

Two independent teams at each site are established to guarantee that the study could be performed in a blinded fashion. The BoNT reconstitution process must be conducted by an unblinded team member. Provided in neutral syringes, solutions of BoNT or saline are not distinguishable from each other, as both having no special colour or smell.

DBS settings are mandatory to be implemented with stimulation amplitude of $0.5 \mathrm{~V}$ below the threshold of possible adverse effects, which was tested before during monopolar review process. This should ensure that patients are not able to perceive switch-on of DBS system.

\section{Summary}

To the best of our knowledge, StimTox-CD is the first randomized, double-blind and controlled comparison of DBS and BoNT in CD. As described, the study protocol is designed to answer the question which therapy method is more effective in controlling motor symptom severity and provides better outcomes concerning disease-related disability and quality of life. The fact that patients will be included in this trial, which have a partial but not satisfactory treatment effect of neurotoxin injections, addresses the unmet medical need of alternative treatment strategies for this less disabled CD population. A positive study outcome would have impact on treatment guidelines for $\mathrm{CD}$. On the condition that DBS proves to be safe in this indication, physicians might be able to offer DBS earlier in the course of $\mathrm{CD}$ and empower patient treatment choices. 


\section{Abbreviations}

AE: adverse events; BDI: Beck Depression Inventory; BoNT: botulinum neurotoxin BPRS: Brief Psychiatric Rating Scale; CD: cervical dystonia; CDQ-24: Craniocervical Dystonia Questionnaire; DBS: deep brain stimulation; EQ-5D: Euroquol (life quality questionnaire); EVA: Evaluation visit; FU: Follow up visit; GDRS: Global Dystonia Rating Scale; GEE: Generalized estimating equation; GPi: Globus pallidus internus; ITT: Intention-to-treat population; MCl: Mild cognitive impairment; MDRS: Mattis Dementia Rating scale; PD: Parkinson's disease; PP: per protocol population; RAN: randomization visit; SCR: screening visit; SD: standard deviation; SF-36: Short Form 36 (life quality questionnaire); TWSTRS: Toronto Western Spasmodic Torticollis Rating Scale

\section{Acknowledgements}

Not applicable.

\section{Funding}

Primary funding of this study was granted by the German Federal Ministry of Education and Research (Bundesministerium für Bildung und Forschung, reference number 01GM1514A), additional funding by an unrestricted grant of MEDTRONIC (Minneapolis, USA). Both institutions had no influence on establishing the study design or writing this manuscript. Collection, analysis and interpretation of data will be run independently from the funding bodies.

\section{Availability of data and materials}

Not applicable.

\section{Authors' contributions}

$\mathrm{JV}, \mathrm{CM}, \mathrm{PUH}$ and UM developed the study design and protocol. PUH and UM were especially responsible for statistical and biometric issues. JV, CM and TMO are active clinical investigators in StimTox-CD. TMO and JV prepared the first draft of the manuscript, which was revised afterwards by $C M$, PUH and UM. All authors read and approved the final manuscript.

\section{Ethics approval and consent to participate}

The protocol conforms to the principles of the declaration of Helsinki and was approved by the ethic committees of each local medical faculty at every study site. The leading ethic committee was located at the Medical Faculty of the University of Wuerzburg (reference number 251/16_ff-sc).

All patients have to give their written consent on study participation. Patients need to be informed about the study in detailed fashion by their treating physician and must obtain sufficient time for consideration. Specific risks of the particular interventions are separately explained by the responsible specialists (BoNT treatment: neurologist, DBS operation: neurosurgeon, general anaesthesia: anaesthesiologist).

\section{Consent for publication}

Not applicable.

\section{Competing interests}

TMO received honoraria from Allergan.

PUH reports grants from German Ministry of Research and Education, German Research Foundation, European Union, Charité-Universitätsmedizin Berlin, Berlin Chamber of Physicians, German Parkinson Society, University Hospital Würzburg, Robert Koch Institute, German Heart Foundation, University Göttingen (within FIND-AF randomized, supported by an unrestricted research grant to the University Göttingen from Boehringer-Ingelheim), University Hospital Heidelberg (within RASUNOA-prime, supported by an unrestricted research grant to the University Hospital Heidelberg from Bayer, BMS, BoehringerIngelheim, Daiichi Sankyo), grants from Charité-Universitätsmedizin Berlin (within Mondafis, supported by an unrestricted research grant to the Charite from Bayer), outside the submitted work.

JV reports consultancy work for Medtronic, Boston Scientific and Newronika. Research grants were provided by Boston Scientific and Medtronic. He received honoraria from Medtronic, Boston Scientific, Newronika, Zambon, Abbott St. Jude, UCB, Desitin, Allergan and IPSEN.

\section{Publisher's Note}

Springer Nature remains neutral with regard to jurisdictional claims in published maps and institutional affiliations.

\section{Author details}

'Department of Neurology, University Hospital Würzburg, Josef-Schneider-Str. 11, 97080 Würzburg, Germany. ${ }^{2}$ Clinical Trial Center, University Hospital Würzburg, 97080 Würzburg, Germany. ${ }^{3}$ Department of Neurosurgery, University Hospital Würzburg, 97080 Würzburg, Germany. ${ }^{4}$ Institute of Clinical Epidemiology and Biometry, University of Würzburg, Comprehensive Heart Failure Center, University of Würzburg, 97080 Würzburg, Germany. ${ }^{5}$ Department of Neurology, University Hospital Würzburg, 97080 Würzburg, Germany.

Received: 21 December 2018 Accepted: 20 January 2019 Published online: 28 February 2019

\section{References}

1. Albanese, A., Bhatia, K., Bressman, S. B., Delong, M. R., Fahn, S., Fung, V. S., Teller, J. K. (2013). Phenomenology and classification of dystonia: A consensus update. Movement Disorders, 28(7), 863-873. doi: https://doi.org/ 10.1002/mds.25475.

2. Benecke, R., Jost, W. H., Kanovsky, P., Ruzicka, E., Comes, G., \& Grafe, S. (2005) A new botulinum toxin type a free of complexing proteins for treatment of cervical dystonia. Neurology, 64(11), 1949-1951. https://doi.org/10.1212/01. WNL.0000163767.99354.C3.

3. Brans, J. W., Lindeboom, R., Snoek, J. W., Zwarts, M. J., van Weerden, T. W., Brunt, E. R., Speelman, J. D. (1996). Botulinum toxin versus trihexyphenidyl in cervical dystonia: A prospective, randomized, double-blind controlled trial. Neurology, 46(4), 1066-1072.

4. Camfield, L., Ben-Shlomo, Y., Warner, T. T., \& Epidemiological Study of Dystonia in Europe Collaborative, G. (2002). Impact of cervical dystonia on quality of life. Movement Disorders, 17(4), 838-841. https://doi.org/10.1002/ mds.10127.

5. Charles, D., Brashear, A., Hauser, R. A., Li, H. I., Boo, L. M., Brin, M. F., \& Group, C. D. S. (2012). Efficacy, tolerability, and immunogenicity of onabotulinumtoxina in a randomized, double-blind, placebo-controlled trial for cervical dystonia. Clinical Neuropharmacology, 35(5), 208-214. https://doi. org/10.1097/WNF.0b013e31826538c7.

6. Comella, C. L., Jankovic, J., Shannon, K. M., Tsui, J., Swenson, M., Leurgans, S., Dystonia Study, G. (2005). Comparison of botulinum toxin serotypes a and B for the treatment of cervical dystonia. Neurology, 65(9), 1423-1429. doi: https://doi.org/10.1212/01.wnl.0000183055.81056.5c

7. Costa, J., Espirito-Santo, C., Borges, A., Ferreira, J. J., Coelho, M., Moore, P., \& Sampaio, C. (2005). Botulinum toxin type a therapy for cervical dystonia. Cochrane Database of Systematic Reviews, 1, CD003633. https://doi.org/10. 1002/14651858.CD003633.pub2.

8. Deuschl, G., Schade-Brittinger, C., Krack, P., Volkmann, J., Schafer, H., Botzel, K., et al. (2006). A randomized trial of deep-brain stimulation for Parkinson's disease. The New England Journal of Medicine, 355(9), 896-908. https://doi. org/10.1056/NEJMoa060281.

9. Jankovic, J., Tsui, J., \& Bergeron, C. (2007). Prevalence of cervical dystonia and spasmodic torticollis in the United States general population. Parkinsonism \& Related Disorders, 13(7), 411-416. https://doi.org/10.1016/j. parkreldis.2007.02.005

10. Kessler, K. R., Skutta, M., \& Benecke, R. (1999). Long-term treatment of cervical dystonia with botulinum toxin a: Efficacy, safety, and antibody frequency. German Dystonia Study Group. Journal of Neurology, 246(4), 265-274.

11. Kimmelman, J., Duckworth, K., Ramsay, T., Voss, T., Ravina, B., \& Emborg, M. E. (2011). Risk of surgical delivery to deep nuclei: A meta-analysis. Movement Disorders, 26(8), 1415-1421. https://doi.org/10.1002/mds.23770.

12. Krauss, J. K., Pohle, T., Weber, S., Ozdoba, C., \& Burgunder, J. M. (1999). Bilateral stimulation of globus pallidus internus for treatment of cervical dystonia. Lancet, 354(9181), 837-838. https://doi.org/10.1016/S01406736(99)80022-1.

13. Kupsch, A., Benecke, R., Muller, J., Trottenberg, T., Schneider, G. H., Poewe, W., et al. (2006). Pallidal deep-brain stimulation in primary generalized or segmental dystonia. The New England Journal of Medicine, 355(19), 19781990. https://doi.org/10.1056/NEJMoa063618.

14. Misra, V. P., Ehler, E., Zakine, B., Maisonobe, P., Simonetta-Moreau, M., \& group, I. I. C. (2012). Factors influencing response to botulinum toxin type a in patients with idiopathic cervical dystonia: Results from an international observational study. BMJ Open, 2(3). https://doi.org/10.1136/bmjopen-2012000881. 
15. Muller, J., Kemmler, G., Wissel, J., Schneider, A., Voller, B., Grossmann, J., et al. (2002). The impact of blepharospasm and cervical dystonia on healthrelated quality of life and depression. Journal of Neurology, 249(7), 842-846. https://doi.org/10.1007/s00415-002-0733-1.

16. Schuepbach, W. M., Rau, J., Knudsen, K., Volkmann, J., Krack, P., Timmermann, L., et al. (2013). Neurostimulation for Parkinson's disease with early motor complications. The New England Journal of Medicine, 368(7), 610-622. https://doi.org/10.1056/NEJMoa1205158.

17. Schuurman, P. R., Bosch, D. A., Bossuyt, P. M., Bonsel, G. J., van Someren, E. J., de Bie, R. M., et al. (2000). A comparison of continuous thalamic stimulation and thalamotomy for suppression of severe tremor. The New England Journal of Medicine, 342(7), 461-468. https://doi.org/10.1056/ NEJM200002173420703.

18. Truong, D., Duane, D. D., Jankovic, J., Singer, C., Seeberger, L. C., Comella, C. L., et al. (2005). Efficacy and safety of botulinum type a toxin (Dysport) in cervical dystonia: Results of the first US randomized, double-blind, placebocontrolled study. Movement Disorders, 20(7), 783-791. https://doi.org/10. 1002/mds.20403.

19. Volkmann, J., Mueller, J., Deuschl, G., Kuhn, A. A., Krauss, J. K., Poewe, W. dystonia, D. B. S. S. g. F. (2014). Pallidal neurostimulation in patients with medication-refractory cervical dystonia: a randomised, sham-controlled trial. Lancet Neurology, 13(9), 875-884. doi: https://doi.org/10.1016/S14744422(14)70143-7.

20. Volkmann, J., Wolters, A., Kupsch, A., Muller, J., Kuhn, A. A., Schneider, G. H. dystonia, D. B. S. S. g. F. (2012). Pallidal deep brain stimulation in patients with primary generalised or segmental dystonia: 5-year follow-up of a randomised trial. Lancet Neurology, 11(12), 1029-1038. doi: https://doi.org/ $10.1016 / 51474-4422(12) 70257-0$

Ready to submit your research? Choose BMC and benefit from:

- fast, convenient online submission

- thorough peer review by experienced researchers in your field

- rapid publication on acceptance

- support for research data, including large and complex data types

- gold Open Access which fosters wider collaboration and increased citations

- maximum visibility for your research: over $100 \mathrm{M}$ website views per year

At $\mathrm{BMC}$, research is always in progress.

Learn more biomedcentral.com/submissions 